\title{
TLR4 Agonist GSK1795091
}

National Cancer Institute

\section{Source}

National Cancer Institute. TLR4 Agonist GSK1795091. NCI Thesaurus. Code C128558.

A toll-like receptor 4 (TLR4) agonist, with potential immunoadjuvant activity. Upon administration, GSK1795091 binds to and activates TLR4, thereby stimulating dendritic cells (DCs), monocytes and macrophages. This activation results in the production of pro-inflammatory cytokines, including interferon gamma (IFN-g), tumor necrosis factoralpha (TNF-a) and the interleukins (IL), IL-1 beta, -6 and -12 . This may induce a T helper cell-1 (Th1) immune response and, upon co-administration of a vaccine containing tumor-associated antigens (TAAs), activates a cytotoxic T-lymphocyte (CTL) response against tumor cells expressing those TAAs. TLR4, a member of the TLR family, plays a key role in the activation of innate immunity. 